\title{
Neutrino scattering on polarized electron target as a test of neutrino magnetic moment
}

\author{
Timur I. Rashba ${ }^{1}$, Victor B. Semikoz \\ The Institute of the Terrestrial Magnetism, the Ionosphere and Radio Wave \\ Propagation of the Russian Academy of Sciences IZMIRAN, Troitsk, Moscow \\ region, 142190, Russia
}

\begin{abstract}
We suggest to use a polarized electron target for improving the sensitivity of the search for the neutrino magnetic moment to the level $\sim 3 \cdot 10^{-13} \mu_{\mathrm{B}}$ in the processes of neutrino (antineutrino) - electron scattering. It is shown that in this case the weak interaction term in the total cross section is significantly suppressed comparing with unpolarized case, but the electromagnetic term does not depend on electron polarization.
\end{abstract}

Key words: neutrino magnetic moment; polarized electron target PACS: $13.10 .+\mathrm{q}, 29.25 . \mathrm{Pj}$

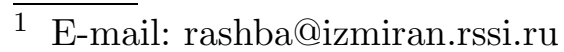

Preprint submitted to Elsevier Preprint 24 October 2018 
The search of new bounds on the neutrino magnetic moment $\mu_{\nu}$ in laboratory experiments would be very important for checking of new Physics beyond the Standard Model (SM) and for different astrophysical implications. The Resonant Spin-Flavor Precession (RSFP) scenario [1,2] in the case of a nonvanishing neutrino magnetic moment $\mu_{\nu} \neq 0$ is still considered as a possible solution to the Solar Neutrino Problem (SNP) [3]. The observations of neutron stars gave an information about the existence of strong magnetic fields (even more than the critical value $B_{\mathrm{c}}=m_{e}^{2} / e=4.41 \cdot 10^{13} \mathrm{G}$ ) which can interact with neutrino through its magnetic moment. The nature of a neutrino (Dirac or Majorana) determines the properties of neutrino magnetic moments, so a Dirac neutrino can have diagonal and transition (off-diagonal) moments, while Majorana neutrinos can have transition moments only [4]. All these hints claim direct laboratory measurements of the neutrino magnetic moment.

There are the laboratory bound on the electron neutrino magnetic moment, $\mu_{\nu_{e}}<1.8 \cdot 10^{-10} \mu_{\mathrm{B}}[5]$, and different astrophysical ones that are even more stringent [6].

To probe neutrino magnetic moment at a more lower level $\mu_{\nu_{e}} \sim 10^{-11} \mu_{\mathrm{B}}$ some artificial neutrino sources of well-known activity and spectra can be used. There are some experiments planned with different possible artificial sources, e.g. ${ }^{51} \mathrm{Cr}[7],{ }^{90} \mathrm{Sr}[8,9],{ }^{147} \mathrm{Pm}[10,11],{ }^{55} \mathrm{Fe}[12]$. The recently proposed measurement of neutrino magnetic moment at a level better than $10^{-11} \mu_{\mathrm{B}}($ $\sim 3 \cdot 10^{-12} \mu_{\mathrm{B}}$ ) with the use of the tritium neutrino emitter (antineutrino source with the energy spectrum endpoint $18.6 \mathrm{keV}$ ) and a semiconductor cryodetector is planned to reach extra low threshold about $1.1 \mathrm{eV} \mathrm{[13].}$

We suggest to use a polarized electron target for more precise measurements of a neutrino magnetic moment in the processes of neutrino (antineutrino) electron scattering. Kinematics of such scattering for which the recoil electron energy $T_{e}=E_{2}-m_{e}$ should be measured is shown in Fig.1. We have fixed $z 0 x$-plane based on the initial neutrino momentum $\vec{k}_{1}$ directed along $z$-axis and on the 3 -vector part $\vec{\xi}_{e}$ of the four-spin $a_{\mu}=\left(0, \vec{\xi}_{e}\right)$ at the rest frame of the initial electron $\left(\vec{p}_{1}=0\right)$ entering the Mishel-Wightman density matrix, $\rho\left(p_{1}\right)=\left(\hat{p}_{1}+m_{e}\right)\left(1+\gamma_{5} \hat{a}\right)$.

The cross section of the electron neutrino (antineutrino) scattering off electrons consists of two terms: weak and electromagnetic ones without interference term that vanishes in the massless limit $m_{\nu} \rightarrow 0$,

$$
\left(\frac{d \sigma}{d T_{e} d \phi}\right)=\left(\frac{d \sigma}{d T_{e} d \phi}\right)^{\text {weak }}+\left(\frac{d \sigma}{d T_{e} d \phi}\right)^{\mathrm{em}} .
$$

After integration over the azimuthal angle $\phi$ (see Fig.1) the weak cross sections have the following forms: 


$$
\begin{aligned}
& \left(\frac{d \sigma}{d T_{e}}\right)_{\nu}^{\text {weak }}=\frac{2 G_{F}^{2} m_{e}}{\pi}\left[g_{e L}^{2}\left(1+\left|\vec{\xi}_{e}\right| \cos \theta_{\xi}\right)+g_{R}^{2}\left(1-\frac{T_{e}}{\omega_{1}}\right)^{2} \times\right. \\
& \left.\times\left(1-\left|\vec{\xi}_{e}\right| \cos \theta_{\xi}\left(1-\frac{m_{e} T_{e}}{\omega_{1}\left(\omega_{1}-T_{e}\right)}\right)\right)-g_{e L} g_{R} \frac{m_{e} T_{e}}{\omega_{1}^{2}}\left(1+\left|\vec{\xi}_{e}\right| \cos \theta_{\xi}\right)\right]
\end{aligned}
$$

for left-handed neutrino and

$$
\begin{aligned}
& \left(\frac{d \sigma}{d T_{e}}\right)_{\tilde{\nu}}^{\text {weak }}=\frac{2 G_{F}^{2} m_{e}}{\pi}\left[g_{R}^{2}\left(1-\left|\vec{\xi}_{e}\right| \cos \theta_{\xi}\right)+g_{e L}^{2}\left(1-\frac{T_{e}}{\omega_{1}}\right)^{2} \times\right. \\
& \left.\times\left(1+\left|\vec{\xi}_{e}\right| \cos \theta_{\xi}\left(1-\frac{m_{e} T_{e}}{\omega_{1}\left(\omega_{1}-T_{e}\right)}\right)\right)-g_{e L} g_{R} \frac{m_{e} T_{e}}{\omega_{1}^{2}}\left(1-\left|\vec{\xi}_{e}\right| \cos \theta_{\xi}\right)\right]
\end{aligned}
$$

for right-handed antineutrino, where $\left|\vec{\xi}_{e}\right|$ is the value of the initial electron polarization. Here $G_{F}$ is the Fermi constant; $g_{e L}=\sin ^{2} \theta_{W}+0.5, g_{R}=\sin ^{2} \theta_{W}$ $\left(\sin ^{2} \theta_{W} \approx 0.23\right)$ are the couplings in the SM; $\omega_{1}=k_{1}$ is the initial neutrino energy; $\theta_{\xi}$ is the angle between the neutrino momentum $\vec{k}_{1}$ and the electron polarization vector $\vec{\xi}_{e}$. If the new (experimental) parameter $\left|\vec{\xi}_{e}\right| \cos \theta_{\xi}$ vanishes, $\left|\vec{\xi}_{e}\right| \cos \theta_{\xi} \rightarrow 0$, our results convert to the standard ones [14].

Electromagnetic terms of the neutrino (antineutrino) scattering cross section do not depend on the electron polarization $\vec{\xi}_{e}$ and have the same form for neutrinos and antineutrinos. If CP holds ${ }^{2}$ the electromagnetic term in Eq. (1) can be written as

$$
\left(\frac{d \sigma}{d T_{e}}\right)^{\mathrm{em}}=\frac{\pi \alpha^{2}}{m_{e}^{2}}\left(\frac{1}{T_{e}}-\frac{1}{\omega_{1}}\right) \frac{\left|\mu_{\nu}\right|^{2}}{\mu_{\mathrm{B}}^{2}},
$$

here $\mu_{\mathrm{B}}$ is the Bohr magneton.

As well as for the case of an unpolarized target $\left(\vec{\xi}_{e}=0\right)$ the electromagnetic interaction via a large magnetic moment increases very significantly in the region of small energy transfer $T_{e}=\omega_{1}-\omega_{2} \ll m_{e}, \omega_{1}$ and can be comparable or greater than the weak interaction. This gives a possibility to determine an upper limit on the neutrino magnetic moment or to measure it.

Weak interaction cross sections (Eqs. (2) and (3)) occur very sensitive both to the polarization of electron target $\left|\vec{\xi}_{e}\right|$ and to the angle $\theta_{\xi}$ between the neutrino

$\overline{2}$ In the Dirac neutrino case when CP conserves the diagonal electric dipole moment is absent, $d_{\nu}=0$. For Majorana neutrino this form is valid if CP parities of initial and final neutrino states are opposite hence the electric dipole moment does not contribute, $d_{i j}=0$ [4]. For the Dirac neutrino transition moment we should substitute $\left|\mu_{\nu}\right|^{2} \rightarrow\left|\mu_{i j}-i d_{i j}\right|^{2}$ where $\mu$ and $d$ are not separated and obey the equality $\operatorname{Re} \mu \operatorname{Re} d+\operatorname{Im} \mu \operatorname{Im} d=0[6]$. 
momentum and the direction of the external magnetic field $\vec{H}$ applied to the detector (= polarization direction, $\vec{H} \| \vec{\xi}_{e}$ ). For the known values of the SM parameters $\left(g_{e L}^{2} \approx 0.533, g_{R}^{2} \approx 0.053\right.$ and $\left.g_{e L} g_{R} \approx 0.168\right)$ one can observe from Eqs. (2) and (3) a new possibility for decreasing of the main weak term $\left(\sim g_{e L}^{2}\right)$ in the cross section Eq. (1) choosing large polarization values $\left(\left|\vec{\xi}_{e}\right| \rightarrow 1\right)$ and with a choice of the specific geometry.

The best case of the antiparallel neutrino (antineutrino) beam $\cos \theta_{\xi}=-1[3$ can be generalized for a more realistic case with an integration over $\theta_{\xi}$ accounting for a concrete geometry of the artificial (isotope) neutrino source placed outside of the detector with known sizes.

Note that the polarization contribution to the $\tilde{\nu} e$-scattering vanishes at the two points:

$$
T_{e 1}=\frac{\omega_{1}^{2}}{m_{e}+\omega_{1}}\left(1+\frac{g_{R}}{g_{e L}}\right), \quad T_{e 2}=\omega_{1}\left(1-\frac{g_{R}}{g_{L}}\right)
$$

For the tritium antineutrino source the polarization reduces (enhances) the unpolarized part of the SM weak cross section below (above) $T_{e 1}$. For the same source the second root $T_{e 2}$ occurs out of the kinematic allowed region, $T_{e 2}>T_{\max }=2 \omega_{1}^{2} /\left(m_{e}+2 \omega_{1}\right)$. Hence in the case of the $\tilde{\nu} e$-scattering the low energy region $T_{e}<T_{e 1}$ is preferable to look for an electromagnetic effect from the sum Eq. (1).

One can easily check that in the case of the $\nu$-scattering $\left(g_{e L} \leftrightarrow g_{R}\right)$ both roots Eq. (5) are out of the kinematic allowed region and the polarization term reduces the SM weak cross-section for any energy $T_{e}$.

Let us consider the spectra of recoil electrons from an antineutrino (neutrino) source calculated via the averaging of the differential cross-sections over the antineutrino (neutrino) spectrum $\rho\left(\omega_{1}\right)$,

$$
\begin{aligned}
S_{\text {free }}^{\mathrm{weak}}\left(T_{e}\right) & =\int_{\omega_{1 \min }} d \omega_{1} \frac{d \sigma^{\mathrm{weak}}\left(T_{e}, \omega_{1}\right)}{d T_{e}} \rho\left(\omega_{1}\right), \\
S_{\text {free }}^{\mathrm{em}}\left(T_{e}\right) & =\int_{\omega_{1 \text { min }}} d \omega_{1} \frac{d \sigma^{\mathrm{em}}\left(T_{e}, \omega_{1}\right)}{d T_{e}} \rho\left(\omega_{1}\right),
\end{aligned}
$$

where weak and electromagnetic cross-sections are given by Eqs. (2), (3) and

$\overline{3}$ This case may be realized for the spherical $4 \pi$-geometry when many magnetized detector crystals are placed on the surface of a sphere and oriented along $\vec{\xi}_{e}$ (with the help of corresponding $\vec{B}$-field solenoids) to the isotope source placed in the center. 
Eq. (4) correspondingly and $\omega_{1 \min }=\left(T_{e} / 2\right)\left[1+\sqrt{1+2 m_{e} / T_{e}}\right]$ is the minimal neutrino energy given by the kinematic limit $T_{e} \leq T_{\max }$.

It was found in [15] that inelastic spectra $S_{\text {in }}(q)$ which depend on the energy transfer $q=\varepsilon_{i}+T_{e}$ for a bound initial electron knocked out from the $i$-shell coincide with the spectra Eq. (6), $S_{\text {in }}(q)=S_{\text {free }}(q) \theta\left(q-\varepsilon_{i}\right)$, when the value $q$ is larger than the binding energy $\varepsilon_{i}, q>\varepsilon_{i}$. Really, $q$ is exactly the event energy measured in the experiment since soft X-quanta and mainly Auger electrons emitted by an atom from which a recoil electron was knocked out are automatically absorbed in the detector fiducial volume or their total energy $\varepsilon_{i}$ adds to the kinetic energy $T_{e}[15]$.

For shells ordered, $i=K, L, M, N, O, \ldots$, let us consider low energy transfer, $\varepsilon_{i-1}>q \geq \varepsilon_{i}$, when antineutrinos (neutrinos) knock out outer (let us say $i=O$, main Bohr number $n=5$ ) electrons only not touching all inner ones. Moreover, since inner electrons from filled shells with $J=L=S=0(i-1=$ $K, L, \ldots)$ do not contribute to the electron polarization one has a sense to consider only the $\nu$-scattering off outer $i$-electrons for which the Zeeman splitting energy is given by

$$
E_{J, M_{J}}=g_{J} \mu_{B} M_{J} H
$$

where $g_{J}$ is the Lande factor and $M_{J}=-J, \ldots, J-1, J$ is the projection of the total spin $\vec{J}$ on the magnetic field direction.

In order to obey such conditions we propose to use a tritium antineutrino source $\left(T_{\max } \approx 1.26 \mathrm{keV}\right)$ and a cryogenic detector with the lowest threshold ( $\sim$ eV-region) for which some lower energy bins $T_{\text {th }} \leq T_{\text {th }}+\Delta T_{e} \leq T_{\text {th }}+$ $2 \Delta T_{e}, \ldots$ within the interval

$$
T_{\mathrm{th}}<q=T_{e}+\varepsilon_{i}<\varepsilon_{i-1}
$$

can be separated. For instance, such low thresholds are considered in [13] for the Si-semiconductor detector without magnetic fields. Hence the recoil electron energy $T_{e}$ should be lower than the difference between the binding energy of the last filled shell $\varepsilon_{i-1}$ and outer (valence) electron energy $\varepsilon_{i}, T_{e}<$ $\varepsilon_{i-1}-\varepsilon_{i}$. In such case only polarized (outer) electrons contribute to the event spectrum to be measured.

One assumes that a low bin width $\Delta T_{e} \sim T_{\text {th }} \sim$ a few $\mathrm{eV}$ would be enough to measure the spectrum $S_{\text {in }}(q)=S_{\text {free }}(q)$.

Note that we have discussed above the spectrum $S_{\text {in }}(q)$ for the ionization process $\nu+e(i) \rightarrow \nu+e$ for an individual electron on the $i$-subshell with a free final electron. Accounting for all polarized electrons on that outer $i$-subshell 
of an atom $Z$ (ion in the detector molecule) we find that the total sum over subshells [15] reduces within the low-energy interval Eq. (8) to the fraction of polarized electrons:

$$
\frac{S_{i n}(q)}{S_{\text {free }}(q)}=\frac{1}{Z} \sum_{i=K, L_{\mathrm{I}-\mathrm{III}} \ldots} n_{i} \theta\left(q-\varepsilon_{i}\right) \rightarrow \frac{n_{O}}{Z}
$$

where $n_{i}$ is the number of electrons on the $i$-subshell, $n_{O}$ is the number of polarized (valence) electrons on the last unfilled, let us say, $i=O$-shell.

The equal reduction of weak and electromagnetic inelastic contributions $S_{i n}(q)$ comparing with the hard energy case $q \gg \varepsilon_{i}$ (for which $S_{\text {in }} \approx S_{\text {free }}$ ) leads to a calculable event statistics decrease. Nevertheless, for low energies Eq. (8) near $T_{\text {th }}$ the conserving ratio

$$
\frac{S_{\text {in }}^{\text {em }}(q)+S_{\text {in }}^{\text {weak }}(q)}{S_{\text {in }}^{\text {weak }}(q)}=\frac{S_{\text {free }}^{\text {em }}(q)+S_{\text {free }}^{\text {weak }}(q)}{S_{\text {free }}^{\text {week }}(q)}
$$

given by Eq. (6) is still more sensitive to the neutrino magnetic moment in the case of polarized outer electrons than for unpolarized targets discussed in [13] (compare in Fig. 2).

The valence electrons can be fully polarized. Really, applying realistic magnetic fields $H \gtrsim 10^{4} \mathrm{G}$ to a cryodetector one can easily reach the ratio $\mu_{\mathrm{B}} H / T>1$ at low temperatures $T \lesssim 0.5 \mathrm{~K}$. Known experimental methods [16] allow to reach high electron polarization at temperatures less than the Curie point $T_{C}$ when the parameter $\mu_{\mathrm{B}} H / T$ is quite large.

For such conditions one can expect $100 \%$ polarization of outer (valence) electrons in the sum over $M_{J}$ entering the electron polarization $\left|\vec{\xi}_{e}\right|=P$,

$$
P=\frac{\sum_{\left|M_{J}\right|}\left[\exp \left(g_{J} \mu_{B}\left|M_{J}\right| H / T\right)-\exp \left(-g_{J} \mu_{B}\left|M_{J}\right| H / T\right)\right]}{\sum_{\left|M_{J}\right|}\left[\exp \left(g_{J} \mu_{B}\left|M_{J}\right| H / T\right)+\exp \left(-g_{J} \mu_{B}\left|M_{J}\right| H / T\right)\right]} .
$$

Here we have used the Boltzman distribution for independent ions (atoms) for which the fraction of the polarized electrons with the total spin projection $M_{J}$ is given by the ratio $N_{M_{J}} / N_{0}=\exp \left(-g_{J} \mu_{B} M_{J} H / T\right)$ where $N_{0}$ is the normalization factor not playing a role in the ratio Eq. (11).

Now let us turn to some experimental prospects. Such weakly interacting Boson gas as atomic hydrogen $H_{4}^{4}$ being spin-polarized in high magnetic fields is used for possible Bose-Einstein condensation at low temperatures [17]

$\overline{4}$ Molecular hydrogen $\mathrm{H}_{2}$ is diamagnetic. 
and seems could be also used as an ideal polarized (100\%) electron target for the $\nu$ e-scattering. However, we do not know how to register recoil electrons there.

The Neganov-Trofimov-Luke effect of ionization-to-heat conversion $[18,19]$ that was proposed for the use of cryogenic semiconductor detectors to measure lowest neutrino magnetic moments $[12,13]$ may be modified for such magnetic semiconductors as ferromagnets $\mathrm{EuS}, \mathrm{EuO}$ where outer $f$-electrons are fully polarized along an external magnetic field [20].

The method should be similar to $[18,19]$ but has some distinctions. At the initial state and low temperatures $T<T_{C}\left(T_{C} \sim 14 \mathrm{~K}\right.$ for $\mathrm{EuS}$ and $T_{C} \sim$ $69 \mathrm{~K}$ for $\mathrm{EuO}$ ) there are no electrons (holes) in conductive (valence) zones, $n_{e}=n_{h} \sim \exp \left(-E_{g} / T\right) \approx 0, E_{g} \gg T$. Then a neutrino hits a polarized valence electron which overcomes the semiconductor gap $E_{g}\left(E_{g}=0.9 \mathrm{eV}\right.$ for $\mathrm{EuO}$ or $E_{g}=1.5 \mathrm{eV}$ for $\mathrm{EuS}$ ) appearing in the conductive zone where it could be accelerated by an external electric field of the known value applied to the crystal to get a measurable energy $\Delta E_{e}$. In the case of a simple semiconductor like Si kept at the thermostat temperature $T$ the following electron-phonon interaction leads to the heating of such Si-crystal for which a low lattice heat capacity $c_{v} \sim\left(T / T_{D}\right)^{3}$ allows to get a large temperature jump $\Delta T \sim \Delta E_{e}\left(T_{D} / T\right)^{3}$ caused by the electron energy absorption $\left(\sim \Delta E_{e}\right)$.

On the other hand, in a ferromagnet semiconductor the lattice heat capacity scales as $c_{v} \sim\left(T / T_{C}\right)^{3 / 2}[20-22]$ where the Curie temperature $T_{C}$ is much lower than the Debye one $T_{D}, T_{C} \ll T_{D}$. As result the electron-magnon interaction heats crystal (through spin-waves exited by a recoil electron) weaker than it happens through phonons in the Si-case. From this we conclude that new cryogenic detectors suggested here at least should be kept at lower temperatures while there are other possibilities that are considered now (e.g. ferromagnet semiconductor $\mathrm{CdCr}_{2} \mathrm{Se}_{4}$ with higher $T_{C} \sim 110 \mathrm{~K}$, etc).

Note that in the low energy transfer region Eq. (8) the weak cross sections in the case of maximal (100\%) electron polarization and for the opposite direction of the magnetic field $\vec{H}$ with respect to the initial neutrino (antineutrino) momentum $\left(\left|\vec{\xi}_{e}\right| \cos \theta_{\xi}=-1\right)$ are 5 times smaller than weak cross sections for the unpolarized case $\left(\left|\vec{\xi}_{e}\right|=0\right)$. The ratios Eq. (10) of the total recoil electron spectra and the weak one for the tritium antineutrino scattering off polarized electrons are shown in Figures 2 and 3.

In a real experiment the parameter $\left|\vec{\xi}_{e}\right| \cos \theta_{\xi}$ may in practice not equal to -1 because of the partially polarized electrons $\left|\vec{\xi}_{e}\right|<1$ as well as due to the non-collinear geometry of the experiment $\left(\theta_{\xi} \neq \pi\right)$. E.g. the ratio of the total cross section and the weak one for $\theta_{\xi} \approx 155$ degrees are shown in Fig. 2 where the polarization parameter $\left|\vec{\xi}_{e}\right| \cos \theta_{\xi}$ is about -0.9 for $\left|\vec{\xi}_{e}\right|=1$. 
Changing the direction of the magnetic field $\vec{H} \rightarrow-\vec{H}$ and then subtracting two corresponding experimental spectra (more correctly subtracting event spectra $\left.N_{\exp }^{ \pm}\left(T_{e}\right) \sim S_{i n}^{ \pm}\left(T_{e}\right)\right)$ one can cancel possible bound electron contributions from unpolarized inner shells + SM weak part in Eq. $(2,3)$ from free electrons + a whole term originated from the electromagnetic cross-section Eq. (4) and a total background.

This would allow us: (i) to separate a pure polarized contribution produced by polarized terms in the weak cross-section Eqs. $(2,3))$ and then (ii) to restore from such net experimental spectrum the total number of polarized electrons contributed to the spectra difference for both magnetic field directions.

Using this number of polarized electrons we could calculate for a given $\vec{H}$ direction the total event number due to the polarized electrons only (adding the corresponding SM part and the electromagnetic term coming from Eq. (4). Then lowering recoil electron energy and comparing this theoretical value $N_{\text {theor }}\left(T_{e}\right)$ with the experimental event number $N_{\exp }\left(T_{e}\right)$ we would be able to control at which $T_{e}$ we could extract the information about an event excess stipulated by corresponding neutrino magnetic moment in the cross-section Eq. (4). The more polarized electrons are involved (when light elements $Z$ are constituents of detector and enter Eq. (9)) the larger statistics and less statistical error will be.

The accuracy of $\mu_{\nu}$-calculations depends also on experimental uncertainties including a background, an isotope source activity, a concrete geometry of the experiment, etc. (see e.g. [9]) that could be done for a future concrete polarized cryodetector with a low threshold and is beyond the scope of the present proposal.

Note that we have omitted radiative corrections $(\mathrm{RC})$ in the main term $g_{e L}^{2}(1+$ $\left.\alpha f_{-} / \pi\right)\left(1+\left|\vec{\xi}_{e}\right| \cos \theta_{\xi}\right)[23]$ entering Eq. (2) where the RC term $\alpha f_{-} / \pi$ is expected at the level $\sim 1 \%$ in the low energy region, or occurs at the negligible level comparing with the strong polarization influence the weak cross-section.

We are grateful to O.G. Miranda, J.W.F. Valle and L.A. Mikaelyan for valuable discussions. We are especially obliged to Prof. E.L. Nagaev for useful consultation concerning magnetic semiconductors. This work was supported in part by the RFBR grant 97-02-16501. 


\section{References}

[1] C.-S. Lim and W.J. Marciano, Phys. Rev. D 37 (1988) 1368.

[2] E. Kh. Akhmedov, Phys. Lett. B 213 (1988) 64.

[3] V. Berezinsky, Talk given at 19th Texas Symposium on Relativistic Astrophysics: Texas in Paris,, France, 14-18 Dec 1998; e-Print Archive: hepph/9904259 (1999).

[4] J.Shechter and J.W.F. Valle, Phys. Rev. D 24 (1981) 1883; D 25 (1982) 283.

[5] A.V.Derbin et al., Yad. Fiz. 57 (1994) 236 [Phys. Atom. Nucl. 57 (1994) 222].

[6] G.G.Raffelt, Stars as Laboratories for Fundamental Physics (The University of Chicago Press, 1996).

[7] N.Ferrari, G.Fiorentini and B.Ricci, Phys. Lett. B 387 (1996) 427.

[8] L.A.Mikaelyan, V.V.Sinev and S.A.Fayans, JETP Lett. 67 (1998) 453.

[9] A.Ianni and D.Montanino, Astropart. Phys. 10 (1999) 331.

[10] V.N.Kornoukhov, Phys. Atom. Nucl. 60 (1997) 558.

[11] I.R. Barabanov et al., Astropart. Phys. 8 (1997) 67.

[12] A.V.Golubchikov, O.A.Zaimidoroga, O.Y.Smirnov and A.P.Sotnikov, Phys. Atom. Nucl. 59 (1996) 1916.

[13] V.N.Trofimov, B.S.Neganov and A.A.Yukhimchuk, Yad. Fiz. 61 (1998) 1373 [Phys. Atom. Nucl. 61 (1998) 1271].

[14] L.B.Okun', Leptons and quarks (Moscow, "Nauka" , 1990).

[15] V.I.Kopeikin, L.A.Mikaelyan, V.V.Sinev and S.A.Fayans, Yad. Fiz. 60 (1997) 2032.

[16] S.R.de Groot, H.A.Tolhoek and W.I.Huiskamp in: Alpha-, beta- and gammaray spectroscopy, edited by K.Siegbahn, Vol. 3 (North-Holland Publishing Company, Amsterdam, 1965).

[17] Isaak F. Silvera, Journal of Low Temperature Physics, 101 (1995) 49.

[18] B.S. Neganov, V.N. Trofimov, URSS patent 1037771 (1981), Otkrytiya, Izobret. 146 (1985) 215.

[19] P. Luke, J. Appl. Phys. 64 (1988) 6858.

[20] E.L. Nagaev, Fizika magnitnykh poluprovodnikov (Moscow, Nauka, 1979), in Russian [E.L. Nagaev, Physics of magnetic semiconductors, translated by M. Samokhvalov, ed. by the author (Mir Publishers, 388 pp., Moscow, 1983)].

[21] J.M. Ziman, Principles of the Theory of Solids (Cambridge, The University Press, 1972), Chapter 10, § 11; 
[22] R.M. White, Quantum Theory of Magnetism ( McGraw-Hill Book Company, New York, 1970), formula (6.64).

[23] J.N.Bahcall, M.Kamionkowski and A.Sirlin, Phys. Rev. D51 (1995) 6146; eprint Arhive: astro-ph/9502003 (1995). 


\section{Figure captions}

Figure 1. Kinematics of the neutrino scattering off the polarized electron.

Figure 2. The ratio of the total recoil electron spectrum and the weak one

(Eq. 10) for different values of the polarization parameter $\left|\vec{\xi}_{e}\right| \cos \theta_{\xi}=0,-0.9,-1$ and for the fixed neutrino magnetic moment $\mu_{\nu}=3 \cdot 10^{-13} \mu_{\mathrm{B}}$. Tritium antineutrino emitter.

Figure 3. The ratio of the total recoil electron spectrum and the weak one (Eq. 10) for different values of the neutrino magnetic moment $\mu_{\nu}=10^{-12} \mu_{\mathrm{B}}$, $3 \cdot 10^{-13} \mu_{\mathrm{B}}, 10^{-13} \mu_{\mathrm{B}}$ and the maximal electron polarization $\left(\left|\vec{\xi}_{e}\right| \cos \theta_{\xi}=-1\right)$. Tritium antineutrino emitter. 


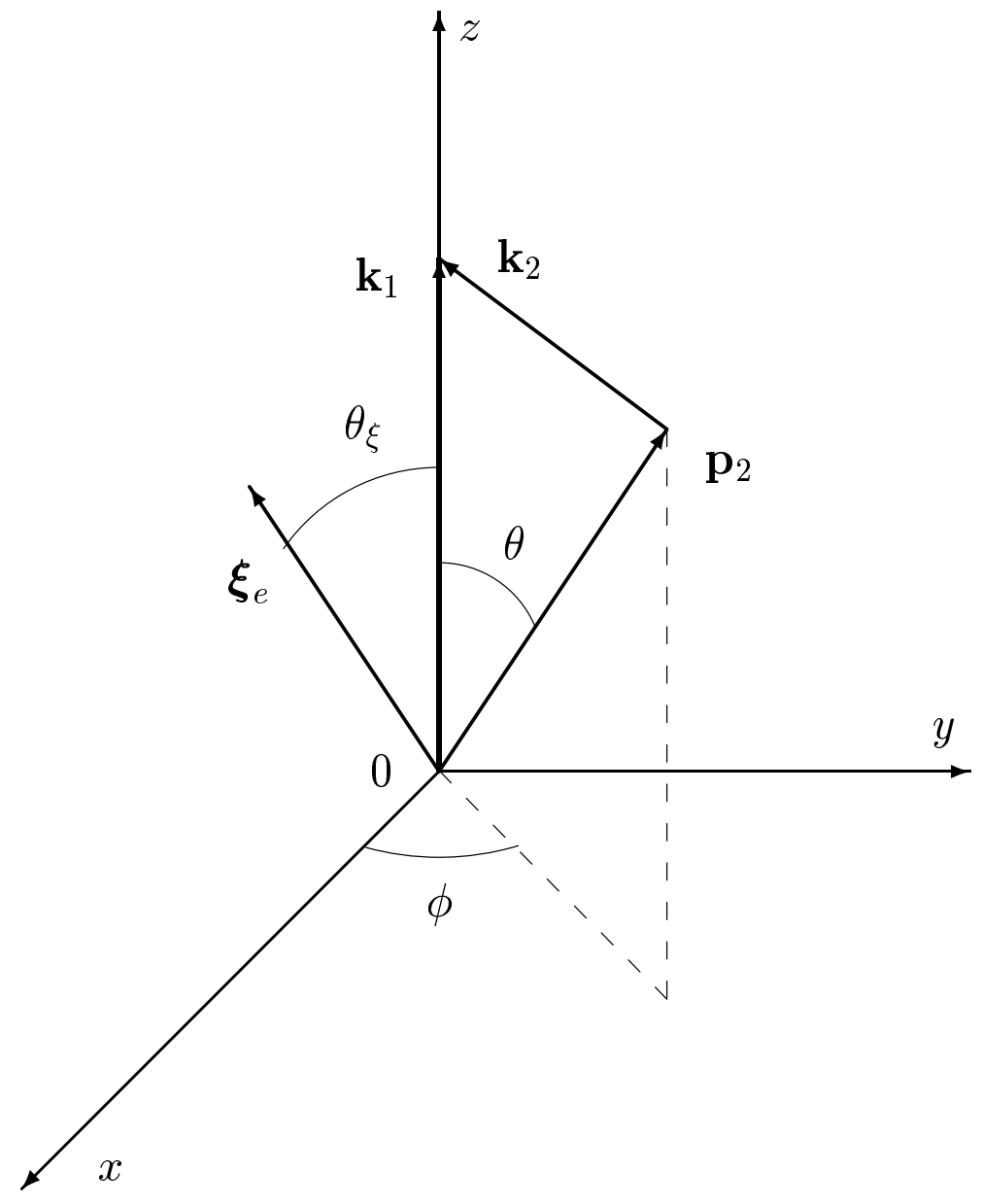

Figure 1 


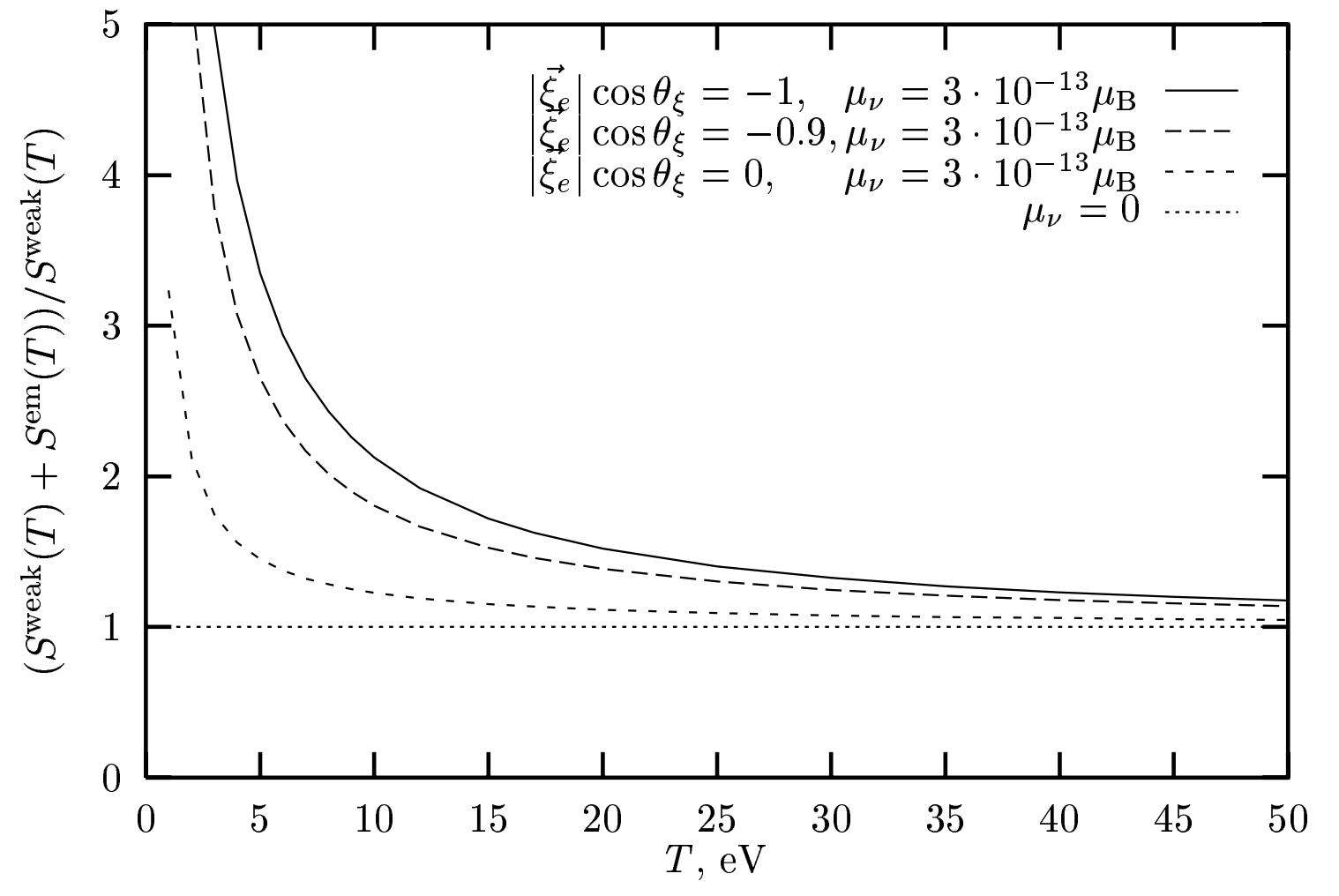

Figure 2 


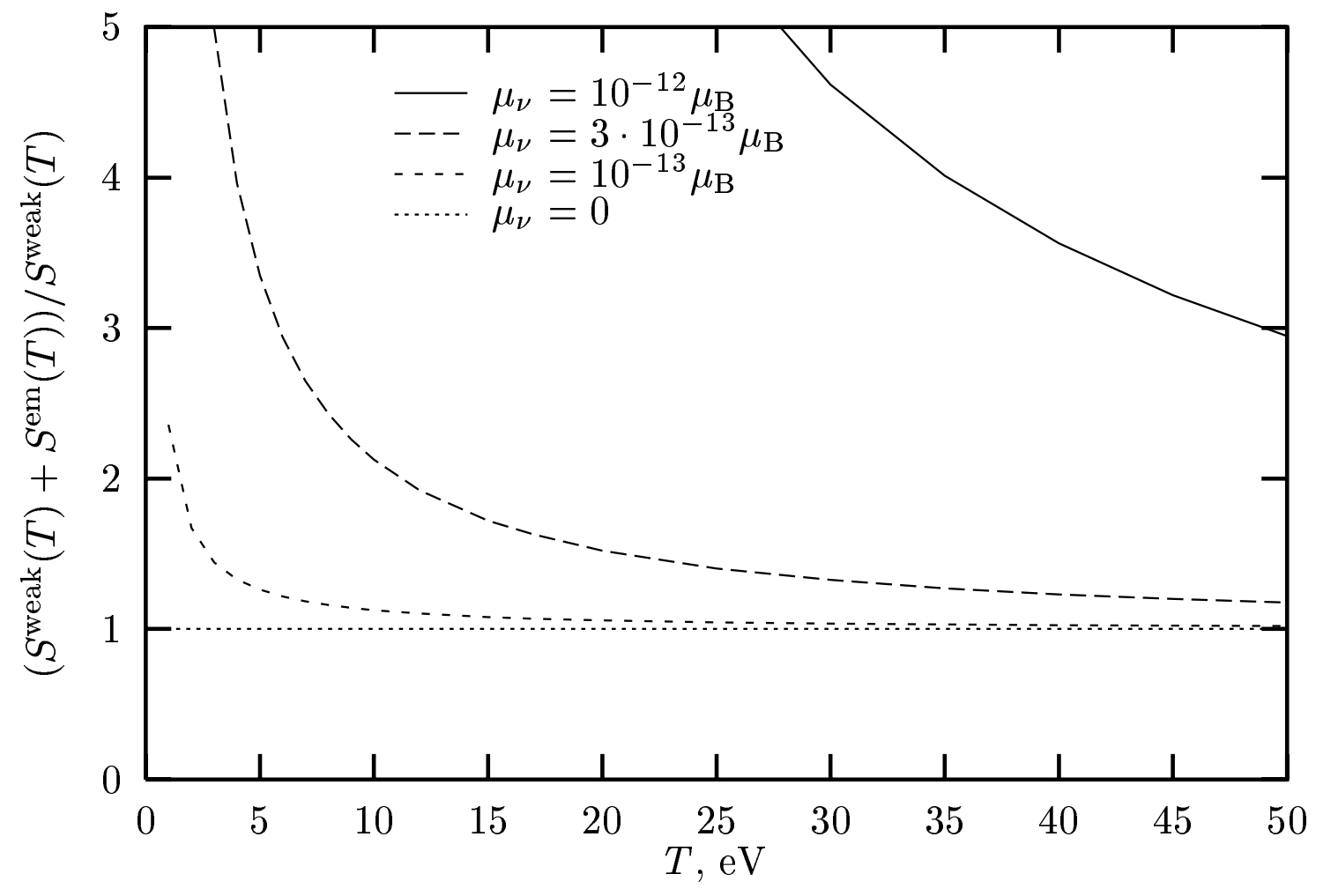

Figure 3 